\title{
BMC
}

Systems Biology

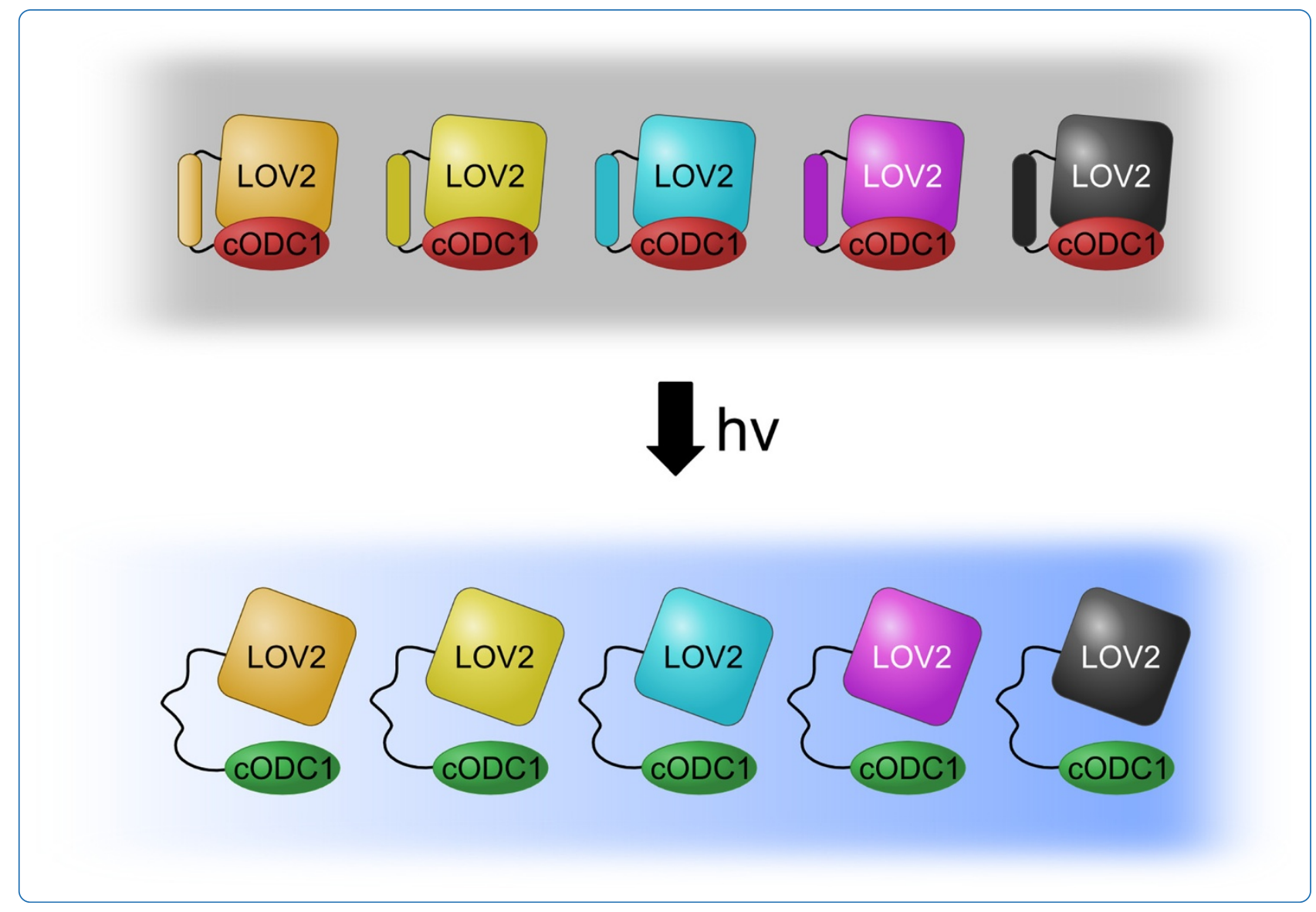

\section{Photo-sensitive degron variants for tuning protein stability by light}

Usherenko et al.

() Biomed Central 


\title{
Photo-sensitive degron variants for tuning protein stability by light
}

Svetlana Usherenko ${ }^{1 \dagger}$, Hilke Stibbe ${ }^{2 \dagger}$, Massimiliano Muscò ${ }^{1}$, Lars-Oliver Essen ${ }^{3}$, Ekaterina A Kostina ${ }^{2}$ and Christof Taxis ${ }^{1 *}$

\begin{abstract}
Background: Regulated proteolysis by the proteasome is one of the fundamental mechanisms used in eukaryotic cells to control cellular behavior. Efficient tools to regulate protein stability offer synthetic influence on molecular level on a selected biological process. Optogenetic control of protein stability has been achieved with the photo-sensitive degron (psd) module. This engineered tool consists of the photoreceptor domain light oxygen voltage 2 (LOV2) from Arabidopsis thaliana phototropin1 fused to a sequence that induces direct proteasomal degradation, which was derived from the carboxy-terminal degron of murine ornithine decarboxylase. The abundance of target proteins tagged with the psd module can be regulated by blue light if the degradation tag is exposed to the cytoplasm or the nucleus.

Results: We used the model organism Saccharomyces cerevisiae to generate psd module variants with increased and decreased stabilities in darkness or when exposed to blue light using site-specific and random mutagenesis. The variants were characterized as fusions to fluorescent reporter proteins and showed half-lives between 6 and 75 minutes in cells exposed to blue light and 14 to 187 minutes in darkness. In blue light, ten variants showed accelerated degradation and four variants increased stability compared to the original psd module. Measuring the dark/light ratio of selected constructs in yeast cells showed that two variants were obtained with ratios twice as high as in the wild type psd module. In silico modeling of photoreceptor variant characteristics suggested that for most cases alterations in behavior were induced by changes in the light-response of the LOV2 domain.
\end{abstract}

Conclusions: In total, the mutational analysis resulted in psd module variants, which provide tuning of protein stability over a broad range by blue light. Two variants showed characteristics that are profoundly improved compared to the original construct. The modular usage of the LOV2 domain in optogenetic tools allows the usage of the mutants in the context of other applications in synthetic and systems biology as well.

Keywords: Optogenetics, LOV2 domain, Degron, Proteolysis, Light, Proteasome, Synthetic biology

\section{Background}

In the last decade, light has been picked up as signal to control cellular behavior taking advantage of natural or engineered photoreceptors that regulate the activity of diverse output domains. This research field is called optogenetics and has recently attracted attention due to the unmatched characteristics of light as signaling entity, mainly the application of light with high temporal and spatial control [1]. The increasing importance of optogenetics for biomedical research is reflected in the

\footnotetext{
*Correspondence: taxis@staff.uni-marburg.de

${ }^{\dagger}$ Equal contributors

'Department of Biology/Genetics, Philipps-Universität Marburg,

Karl-von-Frisch-Strasse 8, 35043 Marburg, Germany

Full list of author information is available at the end of the article
}

development of diverse tools like microbial rhodopsins to control the nervous system of higher eukaryotes, usage of phytochromes and cryptochromes to control transcription in prokaryotes and eukaryotes, light oxygen voltage 2 (LOV2) domain-based control of a small GTPase or a formin, and the usage of the LOV2 domain or phytochromes to control protein localization [2-8]. Especially the LOV2 domain, which originates from plant phototropins, has been selected repeatedly as a tool to establish light-control of protein activity [9-12]. The LOV2 domain consists of a core domain with a Per-ARNT-Sim fold, which binds flavin mononucleotide (FMN) noncovalently as cofactor. An amphipathic helix named the J $\alpha$ helix follows C-terminally to the core domain after a short loop consisting of a few residues [13,14]. The J $\alpha$ helix is 
additionally linked to the core by a series of noncovalent interactions involving hydrophobic as well as polar amino acids [13,15]. Blue-light exposure of a LOV2 domain induces excitation of the FMN cofactor, which leads to the formation of a covalent cysteinyl-flavin $\mathrm{C} 4 \mathrm{a}$ adduct that results in a conformational change of the core domain, detachment of the J $\alpha$ helix from the core and subsequent unfolding of the helix [16]. This light-induced reaction takes place in the Arabidopsis thaliana LOV2 domain on a very short time-scale. The time constants have been measured to be $2 \mu$ s for photon absorption and adduct formation, $1 \mathrm{~ms}$ for the subsequent unfolding of the $\mathrm{J} \alpha$ helix, and about $70 \mathrm{~s}$ for the reversion to the dark state. The latter conversion includes transition of FMN to the ground state and refolding of the J $\alpha$ helix [17]. Dark state reversion varies widely between different LOV domains with timescales spanning from seconds to days [18], which has been in the focus of many studies aiming to uncover the structural features responsible for the differences in reversion kinetics. These efforts led to the identification of several residues in the core domain close to the FMN cofactor that influence reversion to the dark state [19-27]. In addition, the J $\alpha$ helix has been recognized as a region, which is important for the light-reaction of LOV2 domains. Using the Avena sativa LOV2 domain, it was shown that amino acid exchanges within the helix alter the signaling characteristics and affect both, the behavior in darkness as well as the behavior upon blue-light illumination [28,29]. Pseudo-lit-state mutants that show constant J $\alpha$ helix undocking have been obtained by mutating residues in the J $\alpha$ helix and residues near the $\mathrm{N}$-terminus of the LOV2 domain [15,30]. The benefit of these efforts was information how LOV2 domains sense light and respond to it as well as the ability to change the light-response of optogenetic tools based on this widely used domain [24,28]. Recently, the structure of the $A$. thaliana phototropin1 LOV2 domain has been published. Strikingly, the J $\alpha$ helix seems to comprise more residues compared to the homologous LOV2 domain from Avena sativa [14].

Most mutant variants of the LOV2 domain that increase the dissociation of the J $\alpha$ helix from the core domain have been obtained by strategies, which favor the recovery of pseudo-lit state mutants $[15,29,30]$. However, it is desirable to obtain mutants that react profoundly to low amounts of light, but have in darkness a tight association of the helix to the core domain. Such mutants might be less important for constructs inducing site-specific activation of effector proteins on a short time-scale like photo-activatable Rac or photo-activatable formin $[2,8]$, but are certainly interesting for applications that require long exposure to blue light as it is the case in optogenetic control of gene expression or of protein stability [3,31-35] to minimize the possibility of adverse effects due to long blue-light expositions of cells.
Control over protein stability has been achieved with the photo-sensitive degron (psd) module. It allows destabilization of selected proteins upon blue-light illumination by fusing it to the carboxy-terminal end of the target. It is composed of the Arabidopsis thaliana phototropin1 LOV2 domain and a synthetic degradation sequence (degron) called cODC1 that has been derived from the carboxy-terminal degron of murine ornithine decarboxylase (ODC). The ODC degron consists of a stretch of 37 amino acids without secondary structure and a cysteine-alanine motif, which is essential for activity of the degron. The cysteine has been shown to be required for proteasomal association, and has to be 19 amino acids away from the carboxy terminus of the protein [36,37]. In the psd module, a 23 amino acid long synthetic variant of the ODC degron has been fused directly to the end of the LOV2 J $\alpha$ helix. This engineered degron is inactive in darkness due to the helical fold upstream of the cysteine-alanine motif and is activated by J $\alpha$ helix unfolding. The psd module has been used to regulate diverse cellular functions in Saccharomyces cerevisiae by light [35]; a similar construct has been developed recently for usage in higher eukaryotes [31]. Here, we report the generation of photo-sensitive degron module variants that are useful to tune protein stability over a broad range with blue light. We characterized the impact of mutations in the LOV2 domain described in the literature as well as mutations obtained by random mutagenesis. In vivo and in silico characterization of the variants demonstrated that we obtained psd modules with increased degradation rate and higher dark/light ratio. Thus, we obtained psd modules that differ profoundly in their dynamic characteristics and will facilitate light-driven depletion of selected target proteins.

\section{Results and discussion}

Variants of the psd module with changed light-reactivity

We aimed to obtain psd module variants with increased destabilizing activity at a blue light illumination intensity that does not influence the growth rate of yeast cells (Additional file 1: Figure S1A and [35]). Even, YAP1 mutant cells that have been reported to be very light-sensitive [38], are still able to grow under these conditions, although slower than wild type cells (Additional file 1: Figure S1B). Different approaches were taken to obtain improved psd module variants: Firstly, mutations that are known to change the characteristics of homologous LOV domains were selected and introduced into the psd module (Figure 1A). Domain swapping between two closely related LOV2 domains has demonstrated that the J $\alpha$ helix region caused differences in dark-state recovery [25]. Thus, we selected mostly mutants within the J $\alpha$ helix. This approach yielded the mutants V19I, G138A, E139N, and N148E, which correspond to 


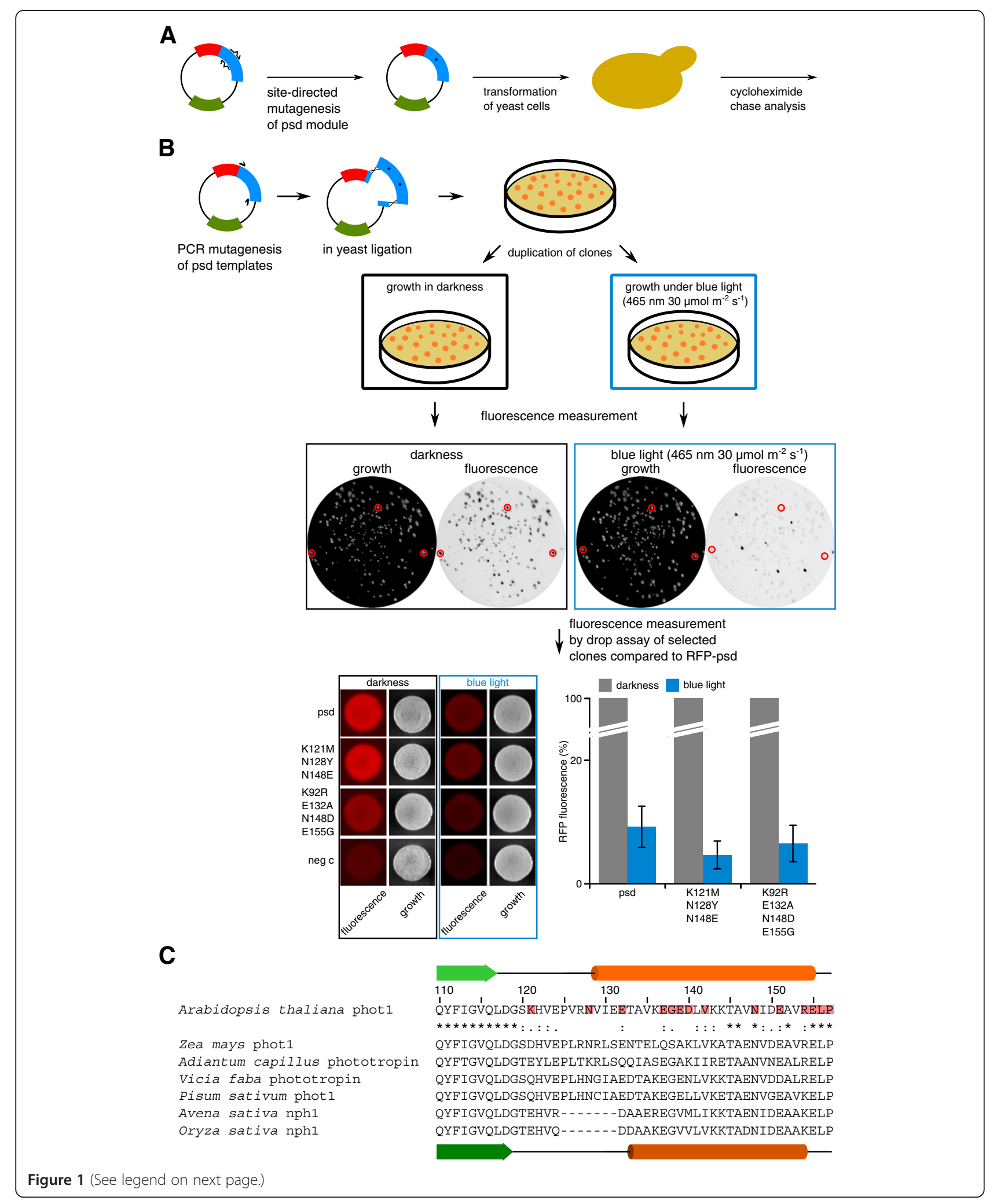


(See figure on previous page.)

Figure 1 Strategy to obtain psd module variants. A) Site-directed mutagenesis was used to generate mutants within the Ja helix. After verification of the construct, yeast cells were transformed with the novel plasmid and subjected to cycloheximide chase analysis. B) Screening procedure to obtain mutants with altered psd module-characteristics. The plasmids pCT337 and pDS91 were used as template during random mutagenesis of the LOV2-CODC1 construct. The PCR products were combined with linearized vector (pDS90) and ligated in yeast by homologous recombination. The yeast clones were grown in darkness on selective solid medium, duplicated and either exposed to blue light or kept in darkness. The RFP fluorescence intensity of each clone was obtained for each condition. Clones with a promising dark/light ratio were selected for patch assays in comparison with the psd module (ESM356-1 + pDS90) as well as a negative control (neg c; ESM356-1 + pRS315) after growth in darkness and under blue light. At least four independent measurements were performed for each clone (lower right graph, error: s.e.m.). C) Sequence alignment of Ja helix-forming residues of LOV2 domains from different phototropins: A. thaliana phot1 (BAD94575.1 residues 569-616), Z. mays phototropin-1 (NP_001147477.1; 476-523), A. capillus phototropin (BAA95669.11:661-708), V. faba phototropin (BAC23098.1; 540-587), P. sativum PsPK4 (AAB41023.2; 542-589), A. sativa nph1 (AAC05083.1; 507-547), and O. sativa nph1 (ABG21841.1; 202-242) were created with the software ClustalX. The secondary structure of $A$. thaliana LOV2 is shown on top (green arrow: $\beta$ strand, orange tube: $\alpha$ helix) and the one of $A$. sativa (dark green arrow: $\beta$ strand, dark orange tube: $a$ helix) below the alignment. The numbering follows the sequence of the psd module. The grade of conservation of an amino acid is indicated using the ClustalX convention. Residues, which are mutated in psd module variants, are indicated by a red box.

the AsLOV2 mutants V416I, G528A, V529N, and N538E $[19,28,29]$. Please note that the numbering of the psd module mutants starts at the methionine of the AtLOV2 domain in the psd module; Additional file 1: Table S1 gives an overview of the mutants and the numbering of the residues in the full length $A$. thaliana phototropin1.

Secondly, a random mutagenesis was performed using the psd module and the N148E variant as templates to create a library of plasmids by in yeast ligation. The N148E mutant was chosen in addition to the wild type psd construct because the corresponding mutant in AsLOV2 (N538E) was reported to have reduced J $\alpha$ helix unfolding in darkness [28], which might be helpful in obtaining a psd module variant with increased dark/light ratio. In these constructs, the red fluorescence protein (RFP) was fused to the amino-terminal end of the psd module as reporter domain. The plasmid library was screened for yeast colonies that showed robust RFP fluorescence after growth in darkness and loss of fluorescence when exposed to blue light. This procedure yielded the mutants V142G, K92R E132A N148D E155G, and K121M N128Y N148E (Figure 1B).

Finally, we created variants by site-directed mutagenesis combining single mutations or changing residues in the Ja helix. Our aim was to destabilize the psd module in blue light without affecting its stability in darkness. Our main criteria was to combine mutations that produced interesting results during the first round of characterization and to generate mutants within the J $\alpha$ helix that disfavor an $\alpha$-helical conformation. For the latter attempt, experimentally obtained helix propensity values were used [39] to select amino acid exchanges that result in altered helix propensity, but do not disturb other characteristics profoundly. We made an alignment of the J $\alpha$ helix region from A. thaliana LOV2 with homologous proteins to indicate all mutagenized residues in relation to conserved features. Moreover, the alignment demonstrates that in phototropins the loop connecting the core domain with the Ja helix is quite variable in sequence
(Figure 1C). Remarkably, this loop of about 11 amino acids $\left(D^{568}\right.$ GSKHVEPVRN ${ }^{578}$ ) is not fully resolved in the structure of the AtLOV2 domain [14].

So far, all the psd module variants contained the synthetic CODC1 sequence, which might differ in its degron activity from the native murine ODC degron. Thus, we created a psd module variant with the last 23 amino acids of the carboxy terminus of murine ornithine decarboxylase (named $\operatorname{deg}_{\mathrm{ODC}}$ ) fused to the AtLOV2 domain instead of the 23 amino acids long synthetic cODC1 sequence used in the psd module. As in the original construct, the unfolding of the LOV2 J $\alpha$ helix is expected to complete the necessary number of unfolded amino acids required to induce proteasomal degradation. In addition, we tried to increase proteasomal association of the psd module upon activation by blue light. To do so, we multiplied the cysteine-alanine motif within the cODC1 sequence to have a double (CACA) or triple (CACACA) motif. In total, we analyzed 24 variants of the psd module for their stability in darkness or under blue light in yeast cells using cycloheximide chase analysis (Figure 2A and Additional file 1: Figure S2A,B). In this assay, the translational inhibitor cycloheximide was used to stop protein synthesis, which allowed us to follow the stability of a selected protein over time by western blotting. We found that the psd module variants were present in yeast cells in good amounts and showed robust light-response, which indicates that the variants were able to fold properly.

\section{Characterization of psd module variants}

We quantified the western blots for each variant and fitted the curves to a first order exponential decay to obtain the half-lives in darkness and blue light (Figure 2B, Table 1, and Additional file 1: Figure S3). The observed half-lives in light ranged from $6.4 \pm 0.2 \mathrm{~min}$ (R154G E155S) to $75 \pm 14 \mathrm{~min}$ (G138A N148E) compared to $20 \pm 1 \mathrm{~min}$ measured for the wild type psd module. In total, 10 of the variants showed a significant reduction in stability and 
A
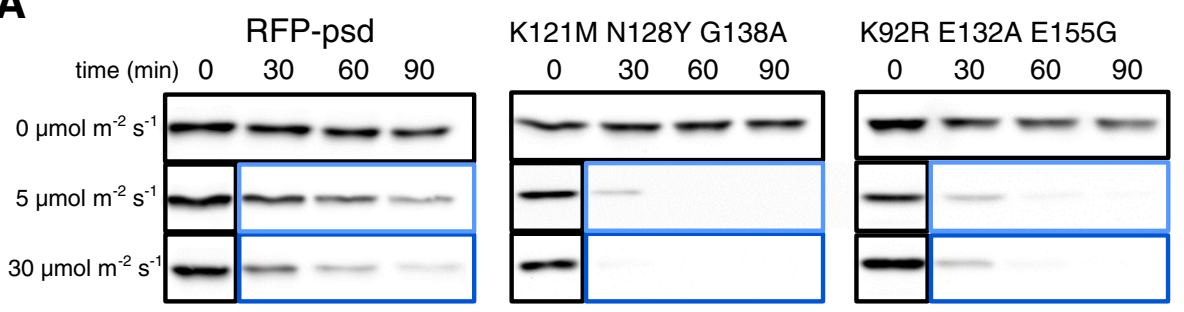

B
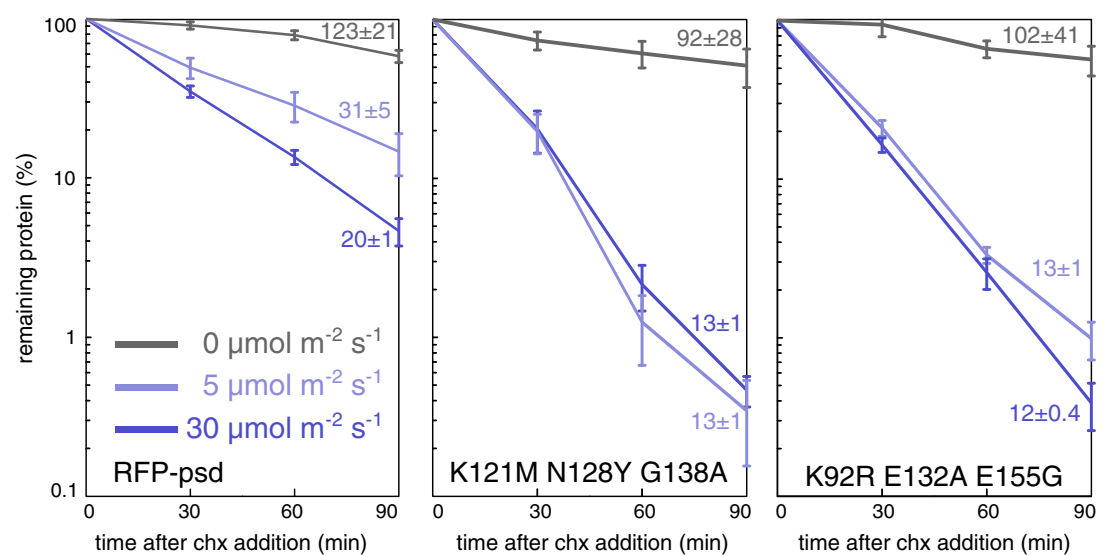

Figure 2 Quantification of psd module variant behavior. A) Yeast cells expressing $P_{A D H 1}$-RFP-psd (plasmid based) or the variants K121M N128Y G138A and K92R E132A E155G were grown in liquid medium in darkness. After removal of the first sample ( $t=0$ hours), cycloheximide (chx) was added to stop protein synthesis; cells were kept in the dark (black box) or exposed to blue light (LED lamp, $465 \mathrm{~nm}$, light blue box: $5 \mu \mathrm{mol} \mathrm{m}^{-2} \mathrm{~s}^{-1}$, dark blue box: $30 \mu \mathrm{mol} \mathrm{m} \mathrm{m}^{-2}$ ) for the rest of the experiment. Equal amounts of sample were collected at the indicated time points and subjected to alkaline lysis and western blotting. B) Quantification of the immunoblots shown in $\mathbf{A}$. Curves are the means obtained from at least four independent measurements (error bars: s.e.m.). The half-lives ( \pm standard error) that are indicated next to each curve were obtained by fitting the data to an exponential decay using the software Origin 7.

four constructs were stabilized compared to the original construct. In darkness, we obtained values from $14 \pm 2 \mathrm{~min}$ (R154G E155S) to $187 \pm 45 \mathrm{~min}(\Delta \mathrm{L} 156$ $\Delta \mathrm{P} 157)$, while we found a half-life of $123 \pm 21 \mathrm{~min}$ in the wild type psd construct. We measured several psd module variants at very low amounts of blue light $\left(5 \mu \mathrm{mol} \mathrm{m}{ }^{-2} \mathrm{~s}^{-1}\right)$ and observed qualitatively similar results (Figure 2A,B, Additional file 1: Figure S2C and S3). For most constructs, the half-lives were somewhat prolonged and ranged from $24 \pm 1$ to $40 \pm 3 \mathrm{~min}$ at $5 \mu \mathrm{mol} \mathrm{m} \mathrm{m}^{-1}$ (31 $\pm 5 \mathrm{~min}$ in psd) compared to 10 to $22 \mathrm{~min}$ at $30 \mu \mathrm{mol} \mathrm{m}{ }^{-2} \mathrm{~s}^{-1}$ in these constructs $(20 \pm 1 \mathrm{~min}$ in psd). However, we identified five mutants that showed almost no difference between the two illumination conditions. We measured half-lives between $9.2 \pm 0.5$ and $13 \pm 1 \mathrm{~min}$ in the K121M N128Y, K92R E132A E155G, G138A V142A R154G E155S, and K121M N128Y G138A variants at $5 \mu \mathrm{mol} \mathrm{m} \mathrm{m}^{-2} \mathrm{~s}^{-1}$ and $8.5 \pm 0.5$ and $13 \pm 1 \mathrm{~min}$ in the same mutants at $30 \mu \mathrm{mol} \mathrm{m} \mathrm{m}^{-1}$. In the V19I variant, we observed have half-lives of $17 \mathrm{~min}$ at both illumination conditions. This mutation corresponds to the A. sativa LOV2 mutation V416I, which has been shown to prolong the dark state recovery time [19]. Thus, the high degradation rate at $5 \mu \mathrm{mol} \mathrm{m}{ }^{-2} \mathrm{~s}^{-1}$ in the variants K121M N128Y,
K92R E132A E155G, G138A V142A R154G E155S, and K121M N128Y G138A might be caused by prolonged dark-state recovery, either induced by changes of the photocycle or extended J $\alpha$ helix refolding time. Remarkably, the psd module variant K121M N128Y has a half-life more than 3 times lower than the original construct at $5 \mu \mathrm{mol} \mathrm{m}{ }^{-2} \mathrm{~s}^{-1}$ (Additional file 1: Figure S3). The four variants K121M N128Y, K92R E132A E155G, G138A V142A R154G E155S, and K121M N128Y G138A were obtained in the second round of psd module variant generation, in which stabilizing and destabilizing mutations were mixed in an attempt to optimize the constructs.

Next, the in vivo ratio of the abundance of the psd module variants in darkness and exposed to blue light was measured. We selected psd module variants with short half-life under blue light and at least moderate stability in darkness. The highest ratios were found in two mutants, K92R E132A E155G and K121M N128Y G138A. For both variants, the ratio was more than two times higher than in the wild type psd module. In the four variants K92R E132A N139E N148D E155G, K121M N128Y, G138A V142A R154G E155S, and N148E R154G E155S, we observed a moderate increase (about 1.4 fold) in the dark/light ratio (Figure 3, Table 1). Interestingly, all 
Table 1 Characteristics of psd module variants

\begin{tabular}{|c|c|c|c|}
\hline Name & Half-life in darkness (min) & $\begin{array}{l}\text { Half-life in } 30 \mu \mathrm{mol} \\
\mathrm{m}^{-2} \mathrm{~s}^{-1} \text { blue light (min) }\end{array}$ & $\begin{array}{l}\text { Dark/light ratio of fluorescence } \\
\text { measurements }\end{array}$ \\
\hline Wild type psd module & $123 \pm 21$ & $20 \pm 1$ & $10.8 \pm 0.5$ \\
\hline V19l & $132 \pm 50$ & $17 \pm 2$ & n.d. \\
\hline K92R E132A E155G & $102 \pm 41$ & $12 \pm 0.4$ & $21.7 \pm 2.5^{*}$ \\
\hline K92R E132A N148D E155G & $103 \pm 26$ & $10.5 \pm 0.3$ & $12.2 \pm 1.1$ \\
\hline K92R E132A E139N N148D E155G & $66 \pm 10$ & $9.8 \pm 0.4$ & $16.3 \pm 1.2^{*}$ \\
\hline K121M N128Y & $44 \pm 8$ & $8.5 \pm 0.3$ & $14.3 \pm 1.4^{*}$ \\
\hline K121M N128Y G138A & $92 \pm 28$ & $13 \pm 1$ & $21.7 \pm 4^{*}$ \\
\hline K121M N128Y N148E & $87 \pm 18$ & $17 \pm 2$ & $9.5 \pm 0.5$ \\
\hline E132D E139K & $89 \pm 25$ & $17 \pm 3$ & n.d. \\
\hline E137D & $165 \pm 43$ & $24 \pm 4$ & n.d. \\
\hline E137D E151D & $79 \pm 25$ & $21 \pm 3$ & n.d. \\
\hline G138A & $147 \pm 52$ & $22 \pm 1$ & n.d. \\
\hline G138A V142A R154G E155S & $42 \pm 10$ & $10.5 \pm 0.8$ & $14.4 \pm 1.4^{*}$ \\
\hline G138A N148E & $151 \pm 53$ & $75 \pm 14$ & n.d. \\
\hline G138A R154G E155S & $27 \pm 4$ & $7 \pm 0.3$ & n.d. \\
\hline E139N & $89 \pm 22$ & $11 \pm 0.5$ & $10.3 \pm 0.4$ \\
\hline V142G & $31 \pm 4$ & $19 \pm 3$ & n.d. \\
\hline N148E & $168 \pm 53$ & $39 \pm 8$ & n.d. \\
\hline N148E R154G E155S & $91 \pm 35$ & $13 \pm 1$ & $13.5 \pm 0.7^{*}$ \\
\hline E151D & $103 \pm 26$ & $28 \pm 6$ & n.d. \\
\hline R154G E155S & $14 \pm 2$ & $6.4 \pm 0.2$ & n.d. \\
\hline$\Delta \mathrm{L} 156 \Delta \mathrm{P} 157$ & $187 \pm 45$ & $52 \pm 9$ & n.d. \\
\hline degodc & $86 \pm 21$ & $20 \pm 3$ & n.d. \\
\hline CACA & $87 \pm 21$ & $20 \pm 3$ & n.d. \\
\hline CACACA & $66 \pm 13$ & $16 \pm 1$ & n.d. \\
\hline
\end{tabular}

The half-lives were obtained by cycloheximide chase analysis and the ratio by fluorescence intensity measurements of cells kept in darkness or exposed to blue light $\left(30 \mu \mathrm{mol} \mathrm{m} \mathrm{m}^{-2} \mathrm{~s}^{-1}\right)$. The asterisk $\left.{ }^{*}\right)$ marks dark/light ratios that are considered to be statistically different from the ratio of psd ( $\left.<<0.05\right)$.

four variants with decreased stability at low illumination intensity (K92R E132A E155G, K121M N128Y, G138A V142A R154G E155S, and K121M N128Y G138A) show also an increase in dark/light ratio. The in vivo measurements highlighted the variants K92R E132A E155G and K121M N128Y G138A, which showed the highest dark/ light switching factor and a faster degradation rate after blue-light illumination than the original construct (Figure 2, Figure 3, and Table 1). These two variants can be expected to be highly useful for in vivo manipulation of protein abundance by light.

The variants with short half-life under blue light showed increased turnover in darkness as well. We measured the impact of the ODC-like degron on protein stability in the variants K92R E132A E155G, K121M N128Y, K121M N128Y G138A, and G138A V142A R154G E155S by mutating the essential cysteine (C160) in the cODC1 degron. This resulted in profound stabilization in all variants under blue light (Additional file 1: Figure S4) and in darkness (data not shown), especially the K121M N128Y G138A variant showed almost complete stabilization. This indicates that the stability in the tested psd module variants depends mostly on cODC1 degron presentation.

Most of the psd module variants that we characterized followed a simple relation: the lower the in vivo stability in darkness, the lower the half-life in cells exposed to blue light (Figure 4). Notably, several psd module variants (G138A, E137D, V19I, N148E, $\Delta$ L156 $\Delta$ P157, G138A N148E) have similar half-lives in darkness (132 to $187 \mathrm{~min}$ ), but different stability under blue light (17 to $75 \mathrm{~min}$ ). This might indicate a very low rate of degron presentation in darkness in these variants, which results in a similar protein turnover rate.

The mutant with the shortest half-life (R154G E155S) does not seem to be particular useful in practical terms due to the very low half-life in darkness. The average half-life of all yeast proteins is $43 \mathrm{~min}$ [40], which means 


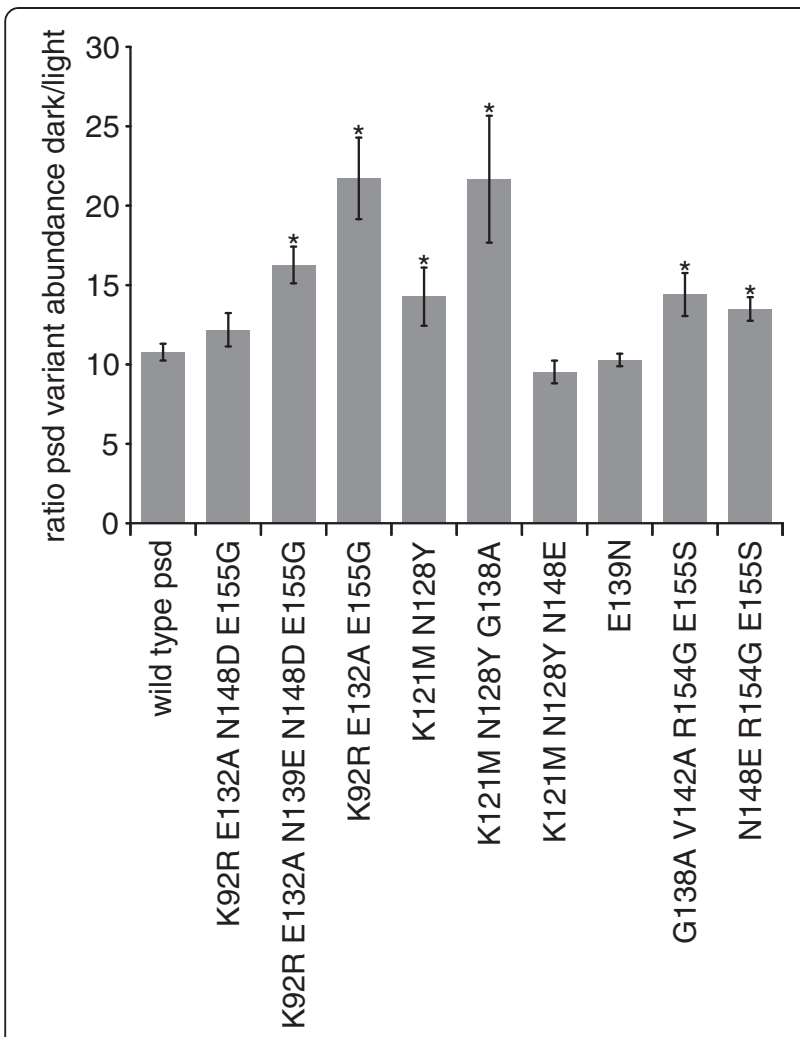

Figure 3 Dark/light ratio of psd module variant abundance. The protein levels of psd module variants were measured in yeast cells grown in darkness or exposed to blue light (LED lamp, $465 \mathrm{~nm}, 30$ $\mu \mathrm{mol} \mathrm{m} \mathrm{s}^{-1}$ ) for 5.5 hours by fluorimeter measurements. Plasmid encoded constructs were used for the in vivo measurements; all variants were derived from the psd module construct $P_{A D H 1}-R F P$-psd. At least six independent measurements were performed for each construct. Error bars: s.e.m.; mean values with a statistically significant difference $(P<0.05)$ to the mean value of the wild type psd module are indicated by an asterisk.

that most yeast proteins would be destabilized already in darkness if tagged with this psd module variant. Also attempts to stabilize this mutant (G138A R154G E155S and G138A V142A R154G E155S) were only partially successful in darkness $(27 \pm 4 \mathrm{~min}$ and $42 \pm 10 \mathrm{~min})$, whereas they retained their short half-life in blue light $(7 \pm 0.3 \mathrm{~min}$ and $10.5 \pm 0.8 \mathrm{~min})$. The triple mutant N148E R154G E155S is affected in darkness and blue light (91 $\pm 35 \mathrm{~min} ; 13 \pm 1 \mathrm{~min}$ ) and has characteristics that are shifted towards the psd module. This series of variants demonstrates that it is possible to tune psd module stability to desired values with appropriate combinations of destabilizing and stabilizing mutations. Similar strategies are feasible for other optogenetic tools using the LOV2 domain as well.

The mutant K121M N128Y, which was derived from a mutant (K121M N128Y N148E) that was picked-up by random mutagenesis, showed a pronounced impact on the stability of the psd module in darkness (half-life $44 \pm 8 \mathrm{~min}$ ) and under blue light $(8.5 \pm 0.3 \mathrm{~min})$. Interestingly, the K121M N128Y mutations map to the loop that connects the J $\alpha$ helix with the LOV2 core domain. This region is very variable among plant LOV2 domains, demonstrated by lack of residue conservation (Figure 1C). Based on statistical coupling analysis, it has been proposed that this loop is a functional sector in the LOV domain family that may induce phenotypic variation [41]. The T517V loop mutation in AsLOV2 showed an unaltered photocycle but increased conformational change upon illumination [42]. It may be of interest to study the impact of mutations in this loop in plant LOV2 domains in detail.

Mutations corresponding to G138A and N148E have been found to increase the dynamic range of the Avena sativa LOV2 domain by stabilizing the docking of the J $\alpha$ helix to the core [28]. We found qualitatively similar effects in the psd module containing the A. thaliana LOV2 domain (Table 1). Especially, the G138A mutation in $A t \mathrm{LOV} 2$ seems to reduce unfolding of the Ja helix in darkness, as has been observed for AsLOV2 [28]. Interestingly, two mutants with very pronounced impact on psd module characteristics had both amino acid exchanges at the C-terminal border of the J $\alpha$ helix. We found strong destabilization for the R154G E155S variant (darkness: $14 \pm 2 \mathrm{~min}$; light: $6.4 \pm 0.2 \mathrm{~min}$; wild type darkness: $123 \pm 21 \mathrm{~min}$; light: $20 \pm 1 \mathrm{~min}$ ) and pronounced stabilization for the $\Delta \mathrm{L} 156 \Delta \mathrm{P} 157$ mutant (darkness: $187 \pm 45 \mathrm{~min}$; light: $52 \pm 9 \mathrm{~min}$ ). This indicates sensitivity of this region towards changes in the amino acid composition. A possible explanation might be that interactions between these residues and amino acids of the core domain stabilize the J $\alpha$ helix in the docked conformation. The crystal structure of AsLOV2 [13] shows that the side chain of L546 is about 4 to $5 \AA$ away from Y508 and F429, which is a distance sufficient for mutual interaction. Strickland and colleagues reported that the AsLOV2 Y508K mutant shows slightly increased undocking of the helix in the dark compared to the wild type [28], whereas an exchange (I428T) in the residue that precedes F429 results in a shortened photocycle lifetime and an increased conformational change [42].

We designed three mutants (E137D, E151D, and E137D E151D) that were expected to destabilize the Ja helix due to changes in helix propensity. We anticipated that this should impact the behavior of the psd module in darkness and in blue light. However, we did not find a pronounced effect on the half-life of the psd module under blue light (Table 1, Additional file 1: Figure S2 and S3). In accordance with this, we did not observe a correlation of helix propensity values with half-life in darkness or under blue light analyzing all psd module variants with mutations in the J $\alpha$ helix (data not shown). This might indicate that electrostatic interactions of the residues between each other and with residues of the core domain are more 


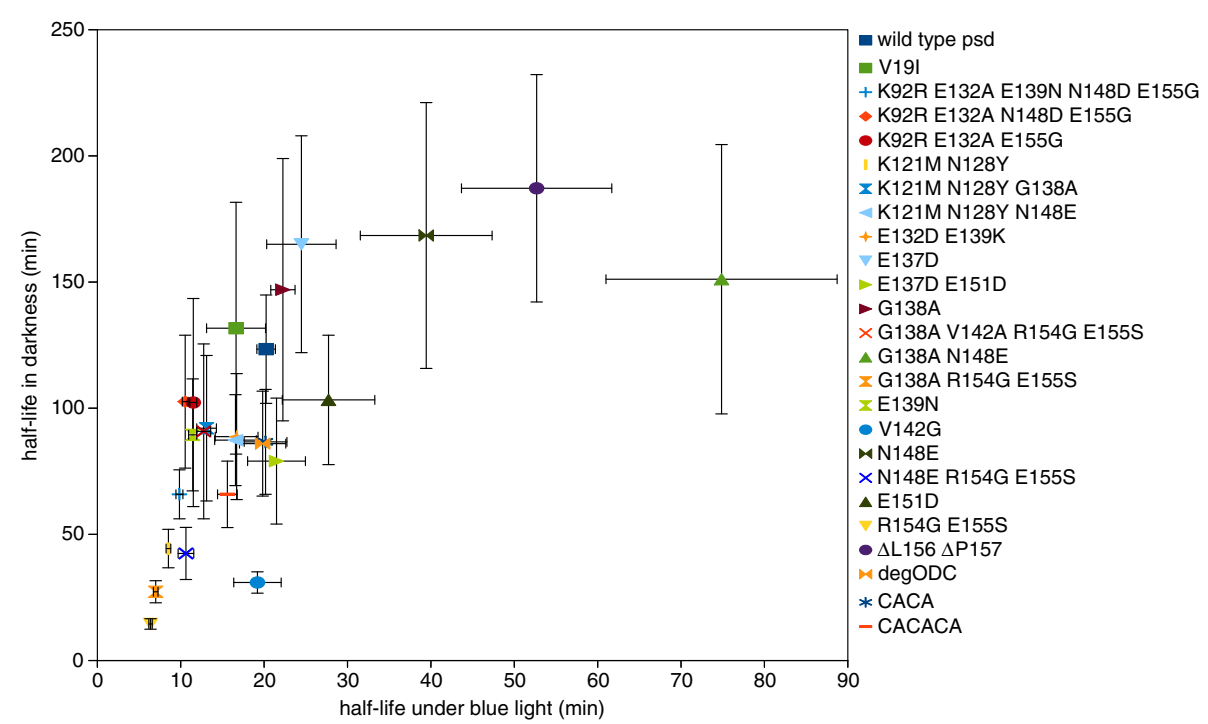

Figure 4 Overview of the characteristics of psd module variants. The half-life in darkness was plotted against the blue-light value for each construct (error bars: standard error).

important for the stability of the helix than the helix propensity values of the helix residues. This is in agreement with findings from analysis of AsLOV2 mutants, which implied that electrostatic interactions are important for the photoresponse of the protein [20]. Site directed exchanges in the degron part of the psd module did not result in a dramatic change of the psd module behavior. The CACACA mutant showed a decrease in half-life to $16 \pm 1 \mathrm{~min}$, whereas the CACA and the deg ODC variants were similar to the wild type psd module $(20 \pm 1 \mathrm{~min})$, although all three mutants showed decreased stability in darkness (Table 1 , Additional file 1: Figure S2 and S3).

\section{Simulation of psd module behavior}

Previously, we used the computer-aided design software TinkerCell [43] to generate a model that simulated the behavior of the psd module [35]. Among other things, the simulation and analysis functions of TinkerCell can be used to predict the steady state levels of proteins within a model or simulate dynamic behavior of a system after an initial change of parameters. The model of the psd module includes a protein synthesis part (pp1) simulating the production of the photo-sensitive degron module protein (PSD), light-driven conversion of the LOV2 dark-state to the lit-state $\left(k_{h v}\right)$, reversion back to the dark state $\left(k_{\text {dark }}\right)$, light-independent conversion to the lit-state $\left(\mathrm{k}_{\text {leak }}\right)$, endogenous protein degradation $\left(k_{\operatorname{degENDO}}\right)$ of PSD, and light-induced protein degradation $\left(\mathrm{k}_{\mathrm{degLOV}}\right)$ of PSD. The differential equations of the model, which we used to simulate psd module behavior, are given in Additional file 1: Figure S5A. In summary, the model simulates the synthesis and degradation of PSD in yeast cells. Comparison of the simulated levels of PSD with in vivo measurements of RFP-psd abundance were in good accordance, the simulations recapitulated quite well the light-response, kinetics of protein depletion, and the difference between abundance in darkness and under blue light illumination [35].

To gain a better quantitative understanding of the novel psd module variants, the conversion constants $k_{\text {dark }}, k_{\text {leak }}, k_{\text {degLOV }}$, and $k_{\text {degENDO }}$ were derived from simulations matching the experimentally derived curves (Additional file 1: Figure S6 and Table S2). We assumed that all mutants have unchanged quantum yields, which was justified by the localization of the mutations that are far from the residues involved in FMN binding. However, we cannot formally exclude that for some mutants the quantum yield might be slightly altered. The experimental data obtained by the cycloheximide chases provided more data points than free parameters in all numerical computations. However the experimental data alone did not guarantee parameter estimates with tight error ranges. A priori information about biological-meaningful parameter ranges was available from the cycloheximide chase experiments (Additional file 1: Figure S3) and literature $[17,29,35-37,44,45]$. According to the Bayesian approach this information must be taken into account. Indeed, a priori information (see Additional file 1: Table S3) together with the experimental data resulted in parameter estimates presented in Additional file 1: Table S2 with tight error ranges. For the psd module itself, changes were only allowed in $\mathrm{k}_{\text {degLOV }}$ and $\mathrm{k}_{\text {degENDO}}$, whereas $\mathrm{k}_{\text {leak }}$ and $k_{\text {dark }}$ were derived from literature [29,35]. Parameter estimation using the curves obtained at light fluxes of 0,5 and $30 \mu \mathrm{mol} \mathrm{m}{ }^{-2} \mathrm{~s}^{-1}$ resulted in values for $k_{\text {degLOV }}$ and $k_{\text {degENDO }}$ very close to previously obtained results 
[35]. We simulated the impact of increased $k_{\text {degLOV }}$ and decreased $k_{\text {dark }}$ in cycloheximide chases compared to simulations with the values obtained for the wild type psd module (Additional file 1: Figure S7A). An increase in $\mathrm{k}_{\text {degLOV }}$ led to slightly faster degradation in darkness and visibly accelerated decay at 5 and $30 \mu \mathrm{mol} \mathrm{m} \mathrm{m}^{-2} \mathrm{~s}^{-1}$ (Additional file 1: Figure $\mathrm{S7B}$ ). This graph resembled the experimental data obtained for the variants K92R E132A N148D E155G or K92R E132A N148D E155G (Additional file 1: Figure S3). A decrease in $\mathrm{k}_{\text {dark }}$ resulted in a graph that had almost no difference between the two illumination conditions and moderate increase in degradation in darkness (Additional file 1: Figure S7C). This is similar to the data obtained from the mutants V19I, K121M N128Y, K121M N128Y G138A, K92R E132A E155G or G138A V142A R154G E155S (Additional file 1: Figure S3).

Simulations with the estimated psd module parameters showed that $67 \%$ of the molecules occupy the lit state at $30 \mu \mathrm{mol} \mathrm{m}{ }^{-2} \mathrm{~s}^{-1}$ blue light, $26 \%$ at $5 \mu \mathrm{mol} \mathrm{m} \mathrm{m}^{-2}$ and $2.5 \%$ in darkness (data not shown). This indicated that at blue light fluxes, which did not affect growth of yeast cells (Additional file 1: Figure S1A and [35]), only a fraction of the molecules is destabilized due to degron activation. The conversion constants obtained from the parameter estimation suggested that for some of the psd module variants a higher lit state fraction might be reached. Examples are the psd module variants V19I, K92R E132A E155G, K121M N128Y, K121M N128Y G138A, and R154G E155S, in which low values for $k_{\text {dark }}$ and $k_{\text {leak }}$ were estimated (Additional file 1: Table S2).

We simulated the behavior of the psd module using the full model, which includes protein production and degradation (Figure 5A). We were interested to model the kinetics of psd module variant depletion at a light flux of $30 \mu \mathrm{mol} \mathrm{m} \mathrm{m}^{-2} \mathrm{~s}^{-1}$. The absolute change of PSD $_{\text {total }}$ numbers was predicted to be higher for the psd module than for most variants with decreased half-life, but these variants reached lower molecule numbers $\left(\mathrm{PSD}_{\text {total }}\right)$ much faster (between 40 and $60 \mathrm{~min}$ ) upon illumination than wild type psd. As expected, slower depletion kinetics were predicted for variants with higher half-life (Figure 5B). In summary, the simulations demonstrated that the novel psd module variants can be used to influence target protein levels by light within a wide range. Most likely, variants with decreased half-life are of greater interest, but the variants give also the possibility to tag two or more target proteins with different psd modules and reach different target protein levels using only one signal.

\section{Conclusions}

Our mutational approach to improve the psd module resulted not only in variants with decreased stability, but
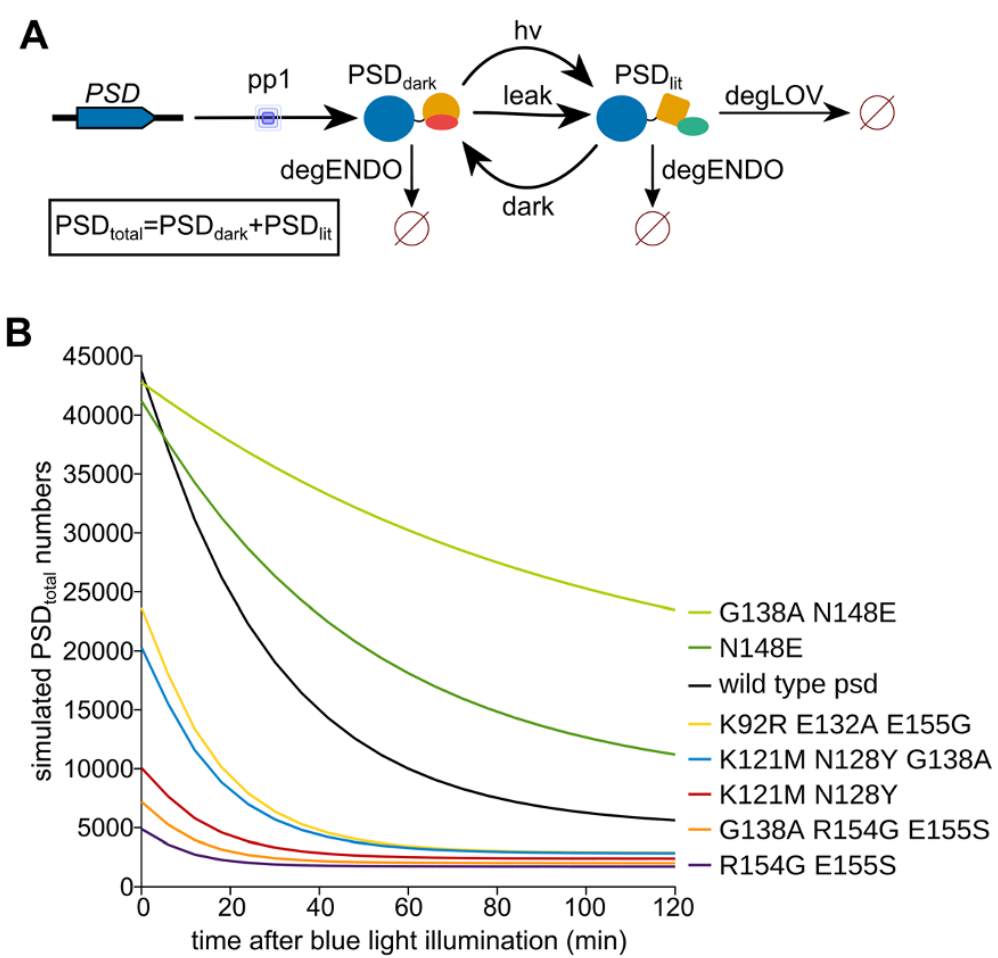

Figure 5 Simulated reaction of psd module variants towards light. A) Model used for the simulations, which contains the protein production module (pp1) as well as the degradation pathways. B) Kinetics of psd module variant depletion. For the simulations, darkness steady state numbers for PSD dark and PSD lit were used as starting values and an exposure to blue light $\left(30 \mu \mathrm{mol} \mathrm{m}{ }^{-2} \mathrm{~s}^{-1}\right)$ was simulated between 0 min and $120 \mathrm{~min}$. 
these variants showed a higher dark/light switching ratio as well. Our characterization of a set of psd module variants and subsequent in silico analysis complements previous studies using the light-regulated trp repressor LovTAP, the photo-controlled kinase YF1, and measurements in single LOV2 domains [21,22,28,42,46].

The novel variants of the photo-sensitive degron module show protein turnover rates that are similar to half-lives that have been measured with other degrons used for induced protein degradation. In yeast, the half-life of murine ODC degron fused to a mutant titin was determined to be about $6 \mathrm{~min}$ [47], similar to the psd module variants with shortest half-life. A faster degradation rate has been measured with an $\mathrm{N}$-degron generated by the ubiquitin fusion technique; an exposed N-terminal arginine induced the degradation of a tester protein with a half-life of about 2 minutes [48]. For the temperature-sensitive degron, which uses an arginine as N-degron as well [49], similar half-lives can be expected in the best case. Another well-developed method is the plant auxin-inducible degron (AID) system for non-plant cells [50]. In yeast, half-lives of about 11 minutes have been determined with this system, measurements in mammalian cells resulted in half-lives between 9 and 18 minutes for different target proteins $[51,52]$. The novel psd module variants reported here showed half-lives that are comparable with the ones of established methods, which are commonly used to degrade target proteins using different signals for induction. The usage of the photo-sensitive degron requires C-terminal fusions to target proteins, which is imposed by the choosen degradation sequence and the mode of activation [35]. Target proteins have to expose the degron to the cytosol or nucleus to be available for regulation by the photo-sensitive degron module, a feature that is shared by all techniques that control protein stability by proteasomal degradation.

Light-mediated regulation has the huge advantage over temperature or chemicals that it can be precisely regulated in quantity, space and time, at least at the level of micro-organisms or cell cultures. A vertebrate-adapted variant of the photo-sensitive degron has been established in zebrafish embryos and mammalian cells, which demonstrates the generality of the method [31]. The continuous illumination that is required to induce depletion of a target protein asks for photo-sensitive degron variants that highly destabilize the target at low illumination strength. The variants we describe here improve the original construct profoundly in these two critical features.

\section{Methods}

Yeast strains and growth conditions

The Saccharomyces cerevisiae strains are derivatives of the S288C strain ESM356-1 (MATa ura3-52 leu2 1 his3 $200 \operatorname{trp1\Delta 63)}$ [53], except for the strains shown in Additional file 1: Figure S1, which are SK1-derivatives (YKS32 (MATa/MATa lys2/lys2 ura3/ura3 leu2/LEU2 ho:: hisG/ho::LYS) [54] and YMM30 (YKS32 yap1A::kanMX/ yap1 $1:$ kanMX)). Standard preparations of media were used for growth on plates [55]. Yeast cells were transformed with plasmids by the lithium acetate method [56]. Low-fluorescence medium $(100 \mathrm{ml}$ salt stock [1\% $\mathrm{KH}_{2} \mathrm{PO}_{4} ; 0.5 \% \quad \mathrm{MgSO}_{4} ; 0.1 \% \mathrm{NaCl} ; 0.1 \% \mathrm{CaCl}_{2} ; 5 \%$ $\left.\left(\mathrm{NH}_{4}\right)_{2} \mathrm{SO}_{4}\right], \quad 0.1 \mathrm{ml}$ trace element stock $[50 \mathrm{mg}$ $\mathrm{H}_{3} \mathrm{BO}_{4} ; 4 \mathrm{mg} \mathrm{CuSO}$; $10 \mathrm{mg} \mathrm{KI} ; 20 \mathrm{mg} \mathrm{FeCl} ; 40 \mathrm{mg}$ $\mathrm{MnSO}_{4} ; 20 \mathrm{mg} \mathrm{Na} \mathrm{MoO}_{4} ; 40 \mathrm{mg} \mathrm{ZnSO}_{4}$ in $100 \mathrm{ml}$ $\left.\mathrm{ddH}_{2} \mathrm{O}\right], 0.1 \mathrm{ml}$ vitamin stock $[0.2 \mathrm{mg}$ biotin; $40 \mathrm{mg}$ calcium pantothenate; $200 \mathrm{mg}$ inositol; $40 \mathrm{mg}$ niacin; $20 \mathrm{mg}$ para-amino benzoic acid; $40 \mathrm{mg}$ pyroxidine $\mathrm{HCl}$;

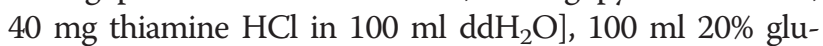
cose, $2 \mathrm{~g}$ of the appropriate amino acid stock in $1 \mathrm{ldd} \mathrm{d}_{2} \mathrm{O}$, sterile filtered) was used to grow yeast cells in liquid cultures in standard plastic cell culture flasks. Blue-light irradiation of yeast cells was performed using custom-build sets of light-emitting diodes (LEDs): high power LED stripes (18 LEDs, $465 \mathrm{~nm}$; revoART, Borsdorf, Germany) or StrawHat LED clusters (6 clusters of 42 LEDs, $465 \mathrm{~nm}$; revoART, Borsdorf, Germany); both sets were equipped with a dimmer to select an appropriate light-flux. For the experiments, light-fluxes of 30 or $5 \mu \mathrm{mol} \mathrm{m} \mathrm{s}^{-1}$ were used, the light-flux was checked before the experiment at the level of the yeast cells (distance yeast cells - LEDs: $10 \mathrm{~cm}$ ) with an optometer (P2000, equipped with light-detector PD-9306-2, Gigahertz-Optik, Türkenfeld, Germany). Yeast cells with and without psd module variants showed no difference in growth in darkness or exposed to a blue-light flux of $30 \mu \mathrm{mol} \mathrm{m}{ }^{-2} \mathrm{~s}^{-1}$ (Additional file 1: Figure S9).

\section{Plasmid construction, random mutagenesis and screening procedure}

Plasmids were constructed by standard procedures [57], details and sequences of the used vectors are available on request; yeast plasmids are listed in Additional file 1: Table S4. The strategy to obtain psd module variants by random mutagenesis is shown in Figure $1 \mathrm{~B}$. The random mutagenesis was performed essentially as described [58] using the template plasmids pCT337 as well as pDS91 and the primers tagrfp_at_lov2_up (AGATATTGTGATTTACCATCTAAATTAGGTCATAAACTGCAGATGAGA AAGGGTATTGATCTAG) and ctermcODC_in_pCT323 downrev (GTGACATAACTAATTACATGACTCGAGT TATTGGAAGTACAAGTTTTCAGAAC). The yeast strain ESM356-1 was transformed with the PCR product together with the linearized plasmid pDS90 (PstI/XmaJI) and grown in darkness on selective solid medium. The clones were duplicated by replica plating, one plate was grown under blue light (465 nm, $30 \mu \mathrm{mol} \mathrm{m} \mathrm{m}^{-2} \mathrm{~s}^{-1}$ ), the other in darkness. The RFP fluorescence intensity of each clone was obtained with the fluorescence image analyzer Fujifilm 
LAS-4000 equipped with a 16-bit CCD-camera, white light (to image growth of yeast cells), green light-emitting LEDs (emission maximum $520 \mathrm{~nm}$ ) and an emission filter set (575 nm-DF20) suitable for RFP observation. The blue light/darkness fluorescence ratio was calculated for each clone and the clones with smallest ratio were selected for further analysis. The selected clones were grown in patches together with the psd module (ESM356-1 + pDS90) in darkness as well as under blue light. After 24 hours, the RFP fluorescence was measured. Plasmids of clones that performed better than the psd module were rescued from yeast cells into $E$. coli by a standard procedure [57] and sequenced.

\section{Cycloheximide chase, immunoblotting, quantitative measurements, sequence alignments, and statistics}

Yeast cells expressing $\mathrm{P}_{A D H 1}$-RFP-psd (plasmid based) or variants thereof were grown in low fluorescence medium in the dark until logarithmic growth phase was reached. The first sample ( $t=0$ hours) was taken and the translation inhibitor cycloheximide (end concentration $200 \mu \mathrm{g} / \mathrm{ml}$ ) was added to stop protein synthesis. Cells were kept in darkness or exposed to blue light for the rest of the experiment. Equal amounts of sample were collected at each time point and subjected to alkaline lysis and western blotting. Immunoblotting experiments were performed as described [58]. Quantification was done with the ImageJ program [59] using the gel analyzer tool. The background in the images was removed with the function $<$ Process $<$ Subtract Background (setting: rolling ball radius 50 pixels). Lifetimes were obtained using the fitting wizard (first order exponential decay) of the program Origin 7 . These were converted into half-lives by multiplying them with the natural logarithm of two. At least four independent measurements were performed for each psd-module variant. The figures show representative results (immunoblotting) or mean results. Error bars show the standard error of the mean (s.e.m.). The flourimeter measurements to obtain dark/light ratios of the RFP-psd variant abundance were essentially done as described [58]. Briefly, logarithmically growing yeast cells (in liquid low fluorescence medium supplemented with $2 \%$ glucose) were exposed to blue light $\left(30 \mu \mathrm{mol} \mathrm{m} \mathrm{m}^{-2} \mathrm{~s}^{-1}\right)$ for 5.5 hours or kept in darkness for the same amount of time. Equal amounts of cells were taken from each condition, treated with sodium azide (10 $\mathrm{mM}$ final concentration), transferred to a black, flat-bottom 96-well microtiter plate (Greiner Bio-One, Germany) and the RFP fluorescence was measured with a microplate reader (Synergy Mx, BioTek, Bad Friedrichshall, Germany). Excitation conditions: 10 flashes of light $(555 \mathrm{~nm})$; fluorescence was observed at a wavelength of $585 \mathrm{~nm}$ with a gain of 130 . Background fluorescence was obtained from yeast cells without construct and subtracted from the measurements of RFP-psd variant containing cells.
The ratio was calculated by dividing the fluorescence intensity measured in darkness with the value obtained from cells exposed to blue light. At least six independent measurements were done for each construct. Statistical analysis (pairwise $t$-test) was done with the QuickCalcs online calculator (www.graphpad.com/quickcalcs/index. $\mathrm{cfm})$. The software ClustalX with standard settings was used to perform sequence alignments [60]. The sequences were obtained from databases maintained by the NCBI (www.ncbi.nlm.nih.gov).

\section{Simulations of cycloheximide chase experiments}

The model used to simulate the behavior of the psd module in cycloheximide chases (Additional file 1: Figure S7) was based on the model for the psd module [35] (shown also in Figure $5 \mathrm{~A}$ ) and was modified with the computeraided design software TinkerCell [43]. The differential equations for cycloheximide chase simulation are shown in Additional file 1: Figure S5B. The protein production module was inactivated $\left(k_{\text {pp1_translation_rate }}=0 \mathrm{~min}^{-1}\right)$ and a fixed number of molecules in the dark and the lit state was used as starting condition. We simulated psd module behavior in cycloheximide chases with the stochastic (exact) analysis function (30 timepoints; 90 minutes). Starting parameters were: $k_{\text {degENDO }}=0.0028 \mathrm{~min}^{-1}$, $\mathrm{k}_{\text {dark }}=0.59 \mathrm{~min}^{-1}, \mathrm{k}_{\mathrm{degLOV}}=0.048 \mathrm{~min}^{-1}, \mathrm{k}_{\mathrm{hv}}=0 \mathrm{~min}^{-1}$ (darkness), $\mathrm{k}_{\mathrm{h} v}=0.2 \mathrm{~min}^{-1}\left(5 \mu \mathrm{mol} \mathrm{m} \mathrm{m}^{-2} \mathrm{~s}^{-1}\right)$ or $\mathrm{k}_{\mathrm{hv}}=$ $1.2 \min ^{-1}\left(30 \mu \mathrm{mol} \mathrm{m} \mathrm{m}^{-2} \mathrm{~s}^{-1}\right), \mathrm{k}_{\text {leak }}=0.01513 \mathrm{~min}^{-1}$, $\mathrm{k}_{\mathrm{pp} 1 \_m R N A \_d e g r a d a t i o n \_r a t e}=0.039 \mathrm{~min}^{-1}$. Initial values were: $\mathrm{PSD}_{\text {dark }}=49000$ molecules, $\mathrm{PSD}_{\text {lit }}=1000$ molecules, pp1_mRNA $=89$ molecules, and psd $=3.3$. The light conversion rate of $k_{\mathrm{hv}}=0.0404 \mathrm{~min}^{-1}$ at a light flux of $1 \mu \mathrm{mol} \mathrm{m} \mathrm{m}^{-2} \mathrm{~s}^{-1}$ was calculated from the quantum yield of 0.26 for FMN [44] multiplied with the FMN cross section of $4.3^{*} 10^{-17} \mathrm{~cm}^{2}$ (at $l_{\max }=450 \mathrm{~nm}$ ) [45] and the number of photons (for a light flux of $1 \mu \mathrm{mol} \mathrm{m}{ }^{-2} \mathrm{~s}^{-1}$, this is $6.023^{*} 10^{13} \mathrm{~cm}^{-2} \mathrm{~s}^{-1}$ ) [35].

Simulations of cycloheximide chase experiments were done for each variant to obtain starting values for parameter estimations (Additional file 1: Table S3). Please note that the half-life in darkness of the psd module and the variants is not solely reflected by $\mathrm{k}_{\text {degENDO }}$ in the model. Rather, unfolding of the J $\alpha$ helix in darkness, which is reflected in the model by $k_{\text {leak }}$, results in cODC1 exposure, which is contributing to the protein degradation in darkness. Furthermore, we use the term half-life throughout the publication to describe the in vivo stability of the psd module and its variants. This should make a clear distinction from the term lifetime, which is commonly used in photobiology to describe the photo-cycle of photoreceptors.

\section{Parameter estimation}

The conversion rates $k_{\text {dark }}, k_{\text {leak }}, k_{\text {degENDO }}$ and $k_{\text {degLOV }}$ for the mutants and the wild type psd module were 
obtained by parameter estimation using the cycloheximide chase data. We estimated parameters by solving the following multiple experiment parameter estimation problem

$$
\min _{y^{k}, k=1, \ldots, N_{\text {exp } p} p} \sum_{k}^{N_{\text {exp }} N_{\text {meas }}} \sum_{j}\left(\frac{H_{j}^{k}-q\left(y^{k}\left(t_{j}\right), p\right)}{\Sigma_{j}}\right)^{2}+\sum_{i=1}^{n_{p}}\left(\frac{p_{i}-p^{\text {apriori }}}{\Sigma}\right)^{2},
$$

$$
\begin{aligned}
\text { s.t. } y^{k} & =f\left(y^{k}, u^{k}, p\right), t \in\left[0, t_{f}\right], y^{k}\left(t_{0}\right)=y_{0}^{k}, \\
k & =1, \ldots, N_{\text {exp }} .
\end{aligned}
$$

Here the dynamics of protein degradation in each experiment was modeled by a system of ordinary differential equations (ODE) (2), where $y^{k} \in \mathbb{R}^{n_{y}}$ denoted the states in the $k$-th experiment at time moment $t, p \in \mathbb{R}^{n_{p}}$ denoted parameters to be estimated and $u_{k}, \quad k=1, \ldots, N_{\exp }$ were controls in each of $N_{\text {exp }}$ experiments. The cost functional (1) described the mismatch between the data $H_{j}^{k}$ and the measurement function. The cost functional also might include a term which characterizes an a priori information on the parameters (Additional file 1: Table S3). These information were obtained from cycloheximide chase simulations with TinkerCell and literature [17,29,35-37,44,45]. For each variant, parameters were chosen that approximated the experimental data. During parameter estimation, these approximations were then used as starting values. In the problem under consideration the states $y(t)$ described the protein concentrations, $y_{1}(t)$ and $y_{2}(t)$ being PSD dark $_{\text {dar }}$ and $\mathrm{PSD}_{\text {lit }}$ concentrations, respectively. The parameters $p_{1}, \ldots, p_{4}$ were $\mathrm{k}_{\text {degENDO}}, \mathrm{k}_{\text {leak }}, \mathrm{k}_{\mathrm{dark}}$ and $\mathrm{k}_{\text {degLOV}}$. The controls $u^{1}=0, u^{2}=0.2$ and $u^{3}=1.2$ described the light intensities in each of the three experiments. In each experiment, we had three measurements at $t_{1}=30$, $t_{2}=60, t_{3}=90$. The measurement function was given by $q(y(t), p)=y_{1}(t)+y_{2}(t)$.

The problem (1)-(2) was solved using the so-called Boundary Value Problem (BVP) Approach for parameter estimation in differential equations. The general idea of the BVP Approach is sketched here, further details of the BVP approach may be found in [61,62]. First a time domain was decomposed and the dynamical model was parametrized by multiple shooting: For an appropriate grid of $M$ time points $T_{j}$

$$
0=T_{1}<T_{2}<\ldots<T_{M}=t_{f},
$$

which covers the measurement interval $\left[0, t_{f}\right]$, the discrete trajectory $s_{j}^{k}:=y^{k}\left(T_{j}\right), \quad k=1, \ldots, N_{\exp } \quad$ was introduced as unknown variables in addition to the unknown parameters $p$. For a given guess for the extended variable vector $\left(s_{1}^{k}, \ldots, s_{M}^{k}, \quad k=1, \ldots, N_{\exp }, p\right)$ the solutions $y^{k}\left(t ; s_{j}^{k}, p\right)$ of the $M-1$ independent initial value problems

$$
\begin{aligned}
\dot{y}^{k} & =f\left(y^{k}, p, u^{k}\right), \\
y^{k}\left(T_{j}\right) & =s_{j}^{k}, \\
t \in I_{j} & :=\left[T_{j}, T_{j+1}\left[, \quad k=1, \ldots, N_{\exp }\right.\right.
\end{aligned}
$$

were calculated on each sub interval $I_{j}$, which resulted in a (usually not continuous) ODE solution. The ODE solution at the measurement points $y^{k}\left(t_{i} ; s_{j(i)}^{k}, u^{k}, p\right)$ for $t_{i} \in\left[T_{j(i)}, T_{j(i)+1}[\right.$ were formally inserted into the cost functional (1). The continuity of the optimal ODE solution was ensured by the following constraints

$$
\begin{aligned}
& y^{k}\left(T_{i+1} ; s_{i}^{k}, p\right)-s_{i+1}^{k}=0, \\
& k=1, \ldots, N_{\exp }, \quad i=0, \ldots, M-1,
\end{aligned}
$$

which were included into the optimization problem.

As a result the parameter estimation problem in the ODE system was transformed into a finite dimensional optimization problem that could be written in the form

$$
\min \frac{1}{2}\left\|F_{1}(x)\right\|_{2}^{2}, \quad \text { s.t. } \quad F_{2}(x)=0 .
$$

Note, that the equalities $F_{2}(x)=0$ included the matching conditions.

This problem was solved by a generalized Gauss-Newton method according to which the new iteration was computed by

$$
x^{k+1}=x^{k}+t^{k} \Delta x^{k}
$$

where the increment $\Delta x^{k}$ solves the quadratic problem

$$
\min _{\Delta x \in \Omega^{k}} \frac{1}{2}\left\|F_{1}\left(x^{k}\right)+\nabla F_{1}\left(x^{k}\right) \Delta x\right\|_{2}^{2}, F_{2}\left(x^{k}\right)+\nabla F_{2}\left(x^{k}\right)^{T} \Delta x=0 .
$$

The linearized problem (3) showed special structures due to multiple experiments and multiple shooting approaches, which were efficiently exploited in a tailored linear algebra method for its solution. With this method the new parameters were computed.

A numerical analysis of the well-posedness of the problem and an assessment of the error of the resulting parameter estimates were performed at the solution $p^{*}$ of the problem (1)-(2). In particular the standard deviations of the parameter estimates were computed.

The parameter estimations were performed with fixed light conversion rates $\left(k_{h v}\right)$ at light fluxes of 0 and $30 \mu \mathrm{mol} \mathrm{m} \mathrm{m}^{-2} \mathrm{~s}^{-1}$ for the mutants and the wild type psd construct. For the wild type psd construct, we allowed a certain variation of $k_{\mathrm{hv}}( \pm 10 \%)$ in case of the light flux of $5 \mu \mathrm{mol} \mathrm{m} \mathrm{m}^{-1} \quad\left(\mathrm{k}_{\mathrm{hv}}=0.379 \pm 0.237 \mathrm{~min}^{-1}\right.$ instead of $\left.0.2 \mathrm{~min}^{-1}\right)$. This did not change the values for the reaction constants $k_{\text {dark }}$, $k_{\text {leak }}, k_{\text {degENDO }}$ and $k_{\text {degLOV }}$ considerably, but lead to much better fitting of the simulated curves to the experimentally derived curves (data not shown). 
This might indicate that illumination conditions were not constant from one experiment to the other during the measurements of the wild type psd module at $5 \mu \mathrm{mol} \mathrm{m} \mathrm{m}^{-2} \mathrm{~s}^{-1}$. For the mutant psd variants, no adjustment of the light conversion rate $k_{h v}$ at $5 \mu \mathrm{mol} \mathrm{m} \mathrm{m}^{-2} \mathrm{~s}^{-1}$ was necessary to obtain good fittings.

The half-life of the K121M N128Y variant exposed to blue light (465 nm, $30 \mu \mathrm{mol} \mathrm{m}{ }^{-2} \mathrm{~s}^{-1}$ ) was used as starting parameter to estimate the $\mathrm{k}_{\text {degLOV }}$ conversion rate ( $0.0564 \mathrm{~min}^{-1} \pm 10 \%$ for most fittings), because this mutant showed the fastest depletion rate of the variants that do not contain a mutation in a region that could influence cODC degron strength. The border was set to residue K143, 37 amino acids away from the carboxy-terminal end of the protein, assuming that only within this region changes might influence degron activity. Thus, the conversion constant $k_{\text {degLOV }}$ was freely adapted, if amino acid exchanges occurred between K143 and the end of the protein.

The conversion rate for endogenous protein turnover $\left(k_{\text {degENDO }}\right)$ was kept fairly constant $(0.0025 \pm 10 \%)$ for all variants assuming that none of the mutations has a considerable impact on protein folding. This was justified by the observation that the variants were detected in good amounts by immunoblotting and showed robust light-reactivity. In addition, we measured the impact of the $\mathrm{CODC} 1$ part by mutating the critical cysteine. This resulted in strong stabilization in all tested cases (Additional file 1: Figure S4). Furthermore, we performed TinkerCell simulations with psd module values increasing only $\mathrm{k}_{\mathrm{degENDO}}$. However, this did not reproduce the experimental data of the variants (data not shown).

Values for $k_{\text {dark }}=0.59 \mathrm{~min}^{-1}$ (70 sec dark recovery time) and $k_{\text {leak }}=0.01513 \mathrm{~min}^{-1}(2.5 \%$ of the molecules in lit state in darkness) $[29,35]$ were used for the psd module and the variants deg ${ }_{\mathrm{ODC}}, \mathrm{CACA}$, and CACACA, in which only the CODC1 part was modified. For the other psd variants, the values for $k_{\text {dark }}$ and $k_{\text {leak }}$ were freely adapted, whereas $k_{\text {degLOV }}$ and $k_{\text {degENDO }}$ were kept within borders as described above. Furthermore, we restricted the values of all conversion rates to exclude solutions with negative parameters. The total amount of PSD $\left(\mathrm{PSD}_{\text {total }}=\mathrm{PSD}_{\text {dark }}+\mathrm{PSD}_{\text {lit }}\right)$ was used to generate graphs.

\section{Simulation of the characteristics of psd module variants}

The full model [35] (also shown in Figure 5A, differential equations Additional file 1: Figure S5A) including protein production and degradation was used to simulate the behavior of the psd variants over time. To do so, $\mathrm{PSD}_{\text {total }}$ numbers were simulated over a time period of $120 \mathrm{~min}$ after light exposure $\left(30 \mu \mathrm{mol} \mathrm{m}{ }^{-2} \mathrm{~s}^{-1}\right)$. First, the molecule numbers for time point 0 were obtained from simulating steady state levels of $\mathrm{PSD}_{\text {dark }}$ and $\mathrm{PSD}_{\text {lit }}$ in darkness for each variant. The $<$ steady state $<$ get state function of TinkerCell was used with following parameters: $k_{\text {dark }}$, $\mathrm{k}_{\text {leak }}, \mathrm{k}_{\mathrm{degENDO}}$ and $\mathrm{k}_{\mathrm{degLOV}}$ as shown in Additional file 1: Table S2 for each variant, $k_{\mathrm{pp} 1 \text { _translation rate }}=2 \mathrm{~min}^{-1}$, $k_{\text {pp1_mRNA_degradation_rate }}=0.039 \mathrm{~min}^{-1}$. Initial values were: pp1_mRNA $=89$ molecules, $\quad$ psd $=3.3$. The obtained values were used as initial values for PSD dark $_{\text {and }}$ $\mathrm{PSD}_{\text {lit }}$ molecule number in the following simulations. There, the $<$ simulation $<$ stochastic (exact) function was used with following parameters: $k_{\text {dark }}, k_{\text {leak }}, k_{\text {degENDO }}$ and $k_{\text {degLOV }}$ as shown in Additional file 1: Table S2 for each variant, $\mathrm{k}_{\mathrm{pp} 1 \text { _translation rate }}=2 \mathrm{~m}^{-1}, \mathrm{k}_{\mathrm{pp} 1 \_\mathrm{mRNA} \_ \text {degradation_rate }}=$ $0.039 \mathrm{~min}^{-1}$. Other initial values: pp1_mRNA $=89$ molecules, psd $=3.3$.

\section{Availability of supporting data}

The data sets supporting the results of this article are included within the article (and its additional file).

\section{Additional file}

Additional file 1: Figure S1. Growth behavior of yeast cells in blue light compared to darkness. Figure S2. Mutational analysis of psd module stability. Figure S3. Quantification of psd module variant behavior. Figure S4. The impact of the cysteine within the CODC1 degron on psd module variant stability. Figure S5. Equations used for the in silico analysis of psd module variants. Figure S6. Parameter estimation to the experimental data of the psd module variants. Figure S7. Influence of increased $k_{\text {degLov }}$ and decreased $k_{\text {dark }}$ on psd module behavior. Figure S8. Comparison of human parameter assumption with parameters obtained by solving the multiple experiment parameter estimation problem. Figure S9. Growth rate measurements of wild type cells and cells expressing psd module variants. Table S1. List of mutated LOV2 domain residues. Table S2. Conversion rate constants of psd module variants obtained by parameter estimation. Table S3. Starting values for parameter estimation. Table S4. Plasmids used in this study. Supplementary references.

\section{Abbreviations}

psd: Photo-sensitive degron; RFP: Red fluorescent protein; FMN: Flavin mononucleotide; LOV: Light oxygen voltage; ODC: Ornithine decarboxylase; LED: Light-emitting diode; ODE: Ordinary differential equation; BVP: Boundary value problem; chx: Cycloheximide.

\section{Competing interests}

The authors declare that they have no competing interests.

\section{Authors' contributions}

SU performed experiments, HS performed parameter estimations, MM provided yeast strains, LOE, EK, and CT discussed results, CT performed experiments, TinkerCell simulations, and wrote the paper. All authors read and approved the manuscript.

\section{Acknowledgements}

We thank R Spadaccini as well as C Renicke for helpful discussions and D Störmer for excellent technical assistance. This work was supported by the DFG grant TA320/3-1, the DFG-funded graduate school GRK1216, and the LOEWE center of Synthetic Microbiology.

\section{Author details}

'Department of Biology/Genetics, Philipps-Universität Marburg, Karl-von-Frisch-Strasse 8, 35043 Marburg, Germany. 'Department of Mathematics and Computer Science/Numerical Optimization, Universität Marburg, Hans-Meerwein-Straße 6, 35032 Marburg, Germany. ${ }^{3}$ Department of Chemistry/Biomedical Research Center, Philipps-Universität Marburg, Hans-Meerwein-Strasse 4, 35032 Marburg, Germany. 
Received: 22 July 2014 Accepted: 30 October 2014

Published online: 18 November 2014

\section{References}

1. Toettcher JE, Voigt CA, Weiner OD, Lim WA: The promise of optogenetics in cell biology: interrogating molecular circuits in space and time. Nat Methods 2011, 8:35-38.

2. Baarlink C, Wang H, Grosse R: Nuclear actin network assembly by formins regulates the SRF coactivator MAL. Science 2013, 340:864-867.

3. Kennedy MJ, Hughes RM, Peteya LA, Schwartz JW, Ehlers MD, Tucker CL: Rapid blue-light-mediated induction of protein interactions in living cells. Nat Methods 2010, 7:973-975.

4. Levskaya A, Chevalier AA, Tabor JJ, Simpson ZB, Lavery LA, Levy M, Davidson EA, Scouras A, Ellington AD, Marcotte EM, Voigt CA: Synthetic biology: engineering Escherichia coli to see light. Nature 2005, 438:441-442.

5. Miesenbock G: Optogenetic control of cells and circuits. Annu Rev Cell Dev Biol 2011, 27:731-758.

6. Shimizu-Sato S, Huq E, Tepperman JM, Quail PH: A light-switchable gene promoter system. Nat Biotechnol 2002, 20:1041-1044.

7. Sorokina O, Kapus A, Terecskei K, Dixon LE, Kozma-Bognar L, Nagy F, Millar AJ: A switchable light-input, light-output system modelled and constructed in yeast. J Biol Eng 2009, 3:15.

8. Wu Yl, Frey D, Lungu Ol, Jaehrig A, Schlichting I, Kuhlman B, Hahn KM: A genetically encoded photoactivatable Rac controls the motility of living cells. Nature 2009, 461:104-108.

9. Lungu OI, Hallett RA, Choi EJ, Aiken MJ, Hahn KM, Kuhlman B: Designing photoswitchable peptides using the AsLOV2 domain. Chem Biol 2012, 19:507-517.

10. Schierling B, Pingoud A: Controlling the DNA cleavage activity of light-inducible chimeric endonucleases by bidirectional photoactivation. Bioconjug Chem 2012, 23:1105-1109.

11. Strickland D, Lin Y, Wagner E, Hope CM, Zayner J, Antoniou C, Sosnick TR, Weiss EL, Glotzer M: TULIPs: tunable, light-controlled interacting protein tags for cell biology. Nat Methods 2012, 9:379-384.

12. Strickland D, Moffat $K$, Sosnick TR: Light-activated DNA binding in a designed allosteric protein. Proc Natl Acad Sci U S A 2008, 105:10709-10714.

13. Halavaty AS, Moffat $\mathrm{K}: \mathrm{N}$ - and C-terminal flanking regions modulate light-induced signal transduction in the LOV2 domain of the blue light sensor phototropin 1 from Avena sativa. Biochemistry 2007, 46:14001-14009.

14. Halavaty AS, Moffat K: Coiled-coil dimerization of the LOV2 domain of the blue-light photoreceptor phototropin 1 from Arabidopsis thaliana. Acta Crystallogr Sect F: Struct Biol Cryst Commun 2013, 69:1316-1321.

15. Harper SM, Christie JM, Gardner KH: Disruption of the LOV-Jalpha helix interaction activates phototropin kinase activity. Biochemistry 2004, 43:16184-16192.

16. Harper SM, Neil LC, Gardner KH: Structural basis of a phototropin light switch. Science 2003, 301:1541-1544.

17. Terazima M: Studies of photo-induced protein reactions by spectrally silent reaction dynamics detection methods: applications to the photoreaction of the LOV2 domain of phototropin from Arabidopsis thaliana. Biochim Biophys Acta 1814, 2011:1093-1105.

18. Zoltowski BD, Gardner KH: Tripping the light fantastic: blue-light photoreceptors as examples of environmentally modulated protein-protein interactions. Biochemistry 2011, 50:4-16.

19. Zoltowski BD, Vaccaro B, Crane BR: Mechanism-based tuning of a LOV domain photoreceptor. Nat Chem Biol 2009, 5:827-834.

20. Zayner JP, Antoniou C, Sosnick TR: The amino-terminal helix modulates light-activated conformational changes in AsLOV2. J Mol Biol 2012, 419:61-74.

21. Christie JM, Corchnoy SB, Swartz TE, Hokenson M, Han IS, Briggs WR, Bogomolni RA: Steric interactions stabilize the signaling state of the LOV2 domain of phototropin 1. Biochemistry 2007, 46:9310-9319.

22. Diensthuber RP, Engelhard C, Lemke N, Gleichmann T, Ohlendorf R, Bittl R, Moglich A: Biophysical, Mutational, and Functional Investigation of the Chromophore-Binding Pocket of Light-Oxygen-Voltage Photoreceptors. ACS Synth Biol 2014. Doi: 10.1021/sb400205x

23. Circolone F, Granzin J, Jentzsch K, Drepper T, Jaeger KE, Willbold D, Krauss $U$, Batra-Safferling R: Structural basis for the slow dark recovery of a full-length LOV protein from Pseudomonas putida. J Mol Biol 2012, 417:362-374.
24. Conrad KS, Bilwes AM, Crane BR: Light-induced subunit dissociation by a light-oxygen-voltage domain photoreceptor from Rhodobacter sphaeroides. Biochemistry 2013, 52:378-391.

25. Jentzsch K, Wirtz A, Circolone F, Drepper T, Losi A, Gartner W, Jaeger KE, Krauss $U$ : Mutual exchange of kinetic properties by extended mutagenesis in two short LOV domain proteins from Pseudomonas putida. Biochemistry 2009, 48:10321-10333.

26. Raffelberg S, Mansurova M, Gartner W, Losi A: Modulation of the photocycle of a LOV domain photoreceptor by the hydrogen-bonding network. J Am Chem Soc 2011, 133:5346-5356.

27. Zoltowski BD, Nash Al, Gardner $\mathrm{KH}$ : Variations in protein-flavin hydrogen bonding in a light, oxygen, voltage domain produce non-Arrhenius kinetics of adduct decay. Biochemistry 2011, 50:8771-8779.

28. Strickland D, Yao X, Gawlak G, Rosen MK, Gardner KH, Sosnick TR: Rationally improving LOV domain-based photoswitches. Nat Methods 2010, 7:623-626.

29. Yao X, Rosen MK, Gardner KH: Estimation of the available free energy in a LOV2-J alpha photoswitch. Nat Chem Biol 2008, 4:491-497.

30. Aihara Y, Yamamoto T, Okajima K, Yamamoto K, Suzuki T, Tokutomi S, Tanaka K, Nagatani A: Mutations in N-terminal flanking region of blue light-sensing light-oxygen and voltage 2 (LOV2) domain disrupt its repressive activity on kinase domain in the Chlamydomonas phototropin. J Biol Chem 2012, 287:9901-9909.

31. Bonger KM, Rakhit R, Payumo AY, Chen JK, Wandless TJ: General method for regulating protein stability with light. ACS Chem Biol 2014, 9:111-115.

32. Konermann S, Brigham MD, Trevino AE, Hsu PD, Heidenreich M, Cong L, Platt R, Scott DA, Church GM, Zhang F: Optical control of mammalian endogenous transcription and epigenetic states. Nature 2013, 500:472-476.

33. Ohlendorf R, Vidavski RR, Eldar A, Moffat K, Moglich A: From dusk till dawn: one-plasmid systems for light-regulated gene expression. J Mol Biol 2012, 416:534-542.

34. Polstein LR, Gersbach CA: Light-inducible spatiotemporal control of gene activation by customizable zinc finger transcription factors. J Am Chem Soc 2012, 134:16480-16483.

35. Renicke C, Schuster D, Usherenko S, Essen LO, Taxis C: A LOV2 domain-based optogenetic tool to control protein degradation and cellular function. Chem Biol 2013, 20:619-626.

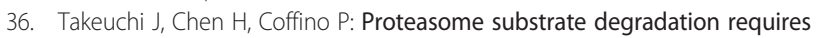
association plus extended peptide. EMBO J 2007, 26:123-131.

37. Takeuchi J, Chen H, Hoyt MA, Coffino P: Structural elements of the ubiquitin-independent proteasome degron of ornithine decarboxylase. Biochem J 2008, 410:401-407.

38. Robertson JB, Davis CR, Johnson CH: Visible light alters yeast metabolic rhythms by inhibiting respiration. Proc Natl Acad Sci U S A 2013, 110:21130-21135.

39. Pace CN, Scholtz JM: A helix propensity scale based on experimental studies of peptides and proteins. Biophys J 1998, 75:422-427.

40. Belle A, Tanay A, Bitincka L, Shamir R, O'Shea EK: Quantification of protein half-lives in the budding yeast proteome. Proc Natl Acad Sci U S A 2006, 103:13004-13009.

41. Halabi N, Rivoire O, Leibler S, Ranganathan R: Protein sectors: evolutionary units of three-dimensional structure. Cell 2009, 138:774-786.

42. Zayner JP, Antoniou C, French AR, Hause RJ Jr, Sosnick TR: Investigating models of protein function and allostery with a widespread mutational analysis of a light-activated protein. Biophys J 2013, 105:1027-1036.

43. Chandran D, Bergmann FT, Sauro HM: TinkerCell: modular CAD tool for synthetic biology. J Biol Eng 2009, 3:19.

44. Drepper T, Eggert T, Circolone F, Heck A, Krauss U, Guterl JK, Wendorff M, Losi A, Gartner W, Jaeger KE: Reporter proteins for in vivo fluorescence without oxygen. Nat Biotechnol 2007, 25:443-445.

45. Islam SDM, Penzkofer A, Hegemann P: Quantum yield of triplet formation of riboflavin in aqueous solution and of flavin mononucleotide bound to the LOV1 domain of Phot1 from Chlamydomonas reinhardtii. Chem Phys 2003, 291:97-114.

46. Gleichmann T, Diensthuber RP, Moglich A: Charting the signal trajectory in a light-oxygen-voltage photoreceptor by random mutagenesis and covariance analysis. J Biol Chem 2013, 288:29345-29355.

47. Henderson A, Erales J, Hoyt MA, Coffino P: Dependence of proteasome processing rate on substrate unfolding. J Biol Chem 2011, 286:17495-17502.

48. Bachmair A, Finley D, Varshavsky A: In vivo half-life of a protein is a function of its amino-terminal residue. Science 1986, 234:179-186. 
49. Dohmen RJ, Wu P, Varshavsky A: Heat-inducible degron: a method for constructing temperature-sensitive mutants. Science 1994, 263:1273-1276.

50. Nishimura K, Fukagawa T, Takisawa H, Kakimoto T, Kanemaki M: An auxin-based degron system for the rapid depletion of proteins in nonplant cells. Nat Methods 2009, 6:917-922.

51. Havens KA, Guseman JM, Jang SS, Pierre-Jerome E, Bolten N, Klavins E, Nemhauser $\mathrm{JL}$ : A synthetic approach reveals extensive tunability of auxin signaling. Plant Physiol 2012, 160:135-142

52. Holland AJ, Fachinetti D, Han JS, Cleveland DW: Inducible, reversible system for the rapid and complete degradation of proteins in mammalian cells. Proc Natl Acad Sci U S A 2012, 109:E3350-E3357.

53. Pereira G, Tanaka TU, Nasmyth K, Schiebel E: Modes of spindle pole body inheritance and segregation of the Bfa1p-Bub2p checkpoint protein complex. EMBO J 2001, 20:6359-6370.

54. Knop M, Strasser K: Role of the spindle pole body of yeast in mediating assembly of the prospore membrane during meiosis. EMBO J 2000, 19:3657-3667.

55. Sherman F: Getting started with yeast. Methods Enzymol 2002, 350:3-41.

56. Schiestl RH, Gietz RD: High efficiency transformation of intact yeast cells using single stranded nucleic acids as a carrier. Curr Genet 1989, 16:339-346.

57. Ausubel FM, Kingston RE, Seidman FG, Struhl K, Moore DD, Brent R, Smith FA (Eds): Current Protocols in Molecular Biology. New York, USA: John Wiley and Sons; 1995

58. Renicke C, Spadaccini R, Taxis C: A tobacco etch virus protease with increased substrate tolerance at the P1' position. PLoS One 2013, 8:e67915.

59. Collins TJ: ImageJ for microscopy. Biotechniques 2007, 43:25-30

60. Thompson JD, Gibson TJ, Plewniak F, Jeanmougin F, Higgins DG: The CLUSTAL_X windows interface: flexible strategies for multiple sequence alignment aided by quality analysis tools. Nucleic Acids Res 1997, 25:4876-4882.

61. Bock HG: Randwertproblemmethoden zur Parameteridentifizierung in Systemen nichtlinearer Differentialgleichungen. Bonn: Universität Bonn; 1987.

62. Bock HG, Kostina E, Schlöder JP: Numerical methods for parameter estimation in nonlinear differential algebraic equations. GAMM-Mitteilungen 2007, 30:376-408.

doi:10.1186/s12918-014-0128-9

Cite this article as: Usherenko et al:: Photo-sensitive degron variants for tuning protein stability by light. BMC Systems Biology 2014 8:128.

\section{Submit your next manuscript to BioMed Central and take full advantage of:}

- Convenient online submission

- Thorough peer review

- No space constraints or color figure charges

- Immediate publication on acceptance

- Inclusion in PubMed, CAS, Scopus and Google Scholar

- Research which is freely available for redistribution 